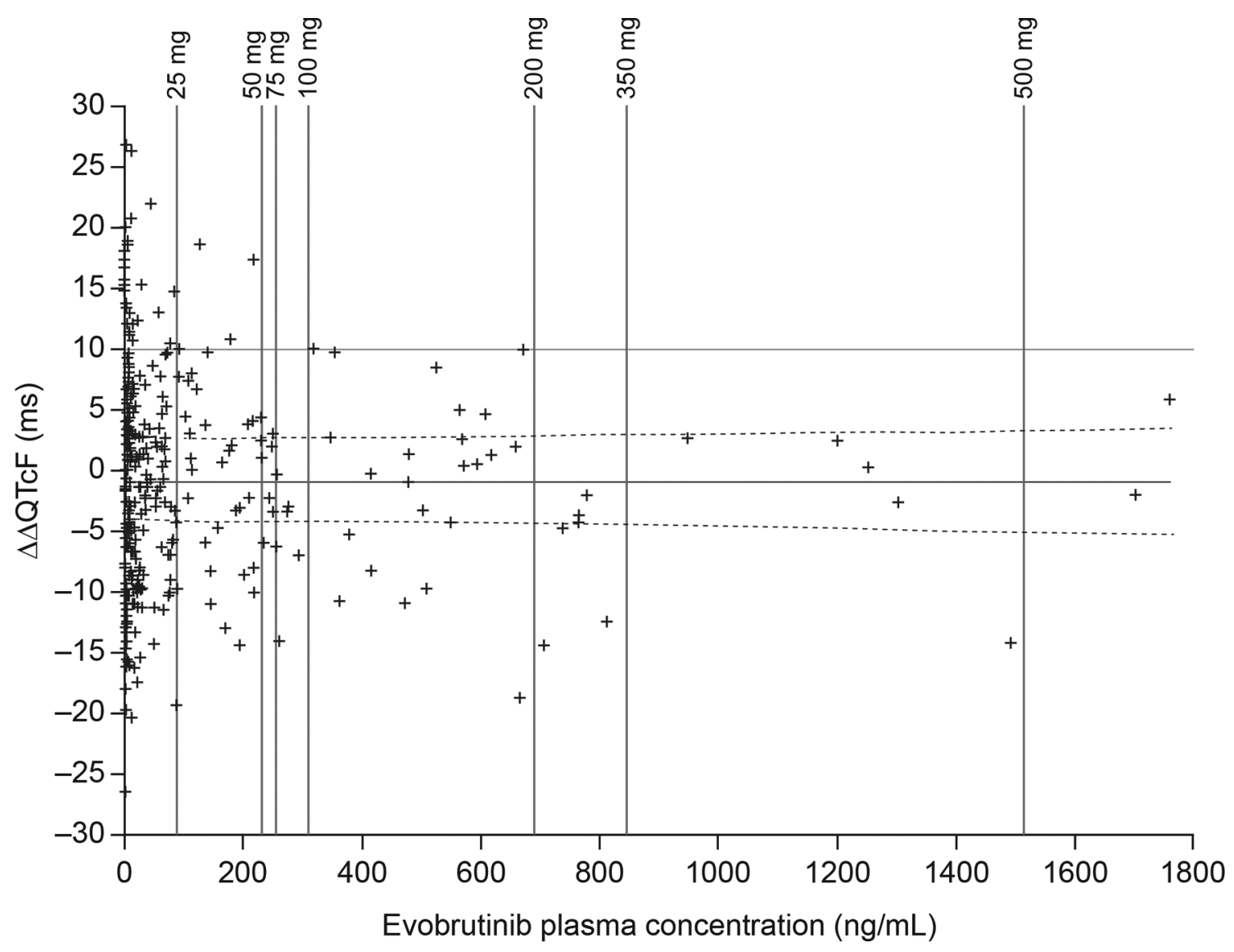

Abstract 215 Figure 1 Relationship between evobrutinib concentration and QTCF

sided bootstrapped confidence interval, which is well below the $10 \mathrm{~ms}$ threshold of regulatory concern (ICH-E14 guidance).

Conclusions Evobrutinib was well-tolerated in healthy volunteers, with predictable PK and no prolongation of QT interval (QTcF). Evobrutinib is undergoing clinical investigation in SLE and other autoimmune diseases.

Funding Source(s): Merck KGaA, Darmstadt, Germany

\section{INHIBITION OF BRUTONS TYROSINE KINASE (BTK) PREVENTS INFLAMMATORY MACROPHAGE DIFFERENTIATION: A POTENTIAL ROLE IN SLE}

${ }^{1}$ Yasemin Beguem Alankus, ${ }^{2}$ Roland Grenningloh, ${ }^{1}$ Philipp Haselmayer, ${ }^{2}$ Andrew Bender, ${ }^{1}$ Julia Bruttger. ${ }^{1}$ Merck KGaA, Darmstadt, Germany; ${ }^{2} E M D$ Serono Research and Development Institute, Inc., Billerica, MA, USA

10.1136/lupus-2019-Ism.216

Background Brutons tyrosine kinase (BTK) mediates B cell receptor $(\mathrm{BCR})$ and $\mathrm{Fc}$ receptor $(\mathrm{FcR})$ signaling in several hematopoietic cell lineages, including $\mathrm{B}$ cells, macrophages and neutrophils. The BTK inhibitor evobrutinib silences B cells and prevents innate immune activation via FcR and has been shown to be efficacious in a preclinical model for SLE. Macrophages can have pro-inflammatory and anti-inflammatory properties and thus they play a crucial role in exacerbation versus control of autoimmune disease. BTK function has been implied downstream of certain cytokine receptors that control macrophage differentiation. The aim of this preclinical study was to investigate the effect of BTK inhibition on the differentiation and activation of monocytes and macrophages.

Methods Monocytes were isolated from the peripheral blood of healthy volunteers. BTK activation was analyzed by Western blot following a $30 \mathrm{~min}$ BTK inhibitor treatment and a subsequent granulocyte-macrophage colony-stimulating factor (GM-CSF) stimulation time course. Survival of GM-CSF differentiated M1 cells was analyzed by flow cytometry following AnnexinV/PI staining. Expression levels of interleukin (IL) $-1 \beta$ and IL-10 were determined by quantitative polymerase chain reaction following 48 hours of GM-CSF stimulation and BTK inhibitor treatment. Tumor necrosis factor alpha (TNF-) levels in cell culture supernatants were measured by ELISA following overnight lipopolysaccharide stimulation and BTK inhibitor treatment. The uptake of apoptotic cells by M2 macrophages was analyzed by flow cytometry.

Results BTK was activated downstream of the GM-CSF receptor. In line with this finding, in vitro GM-CSF differentiated M1 macrophages underwent apoptosis upon BTK inhibition using evobrutinib. Monocytes treated with GM-CSF in the presence of BTK inhibitor secreted less TNF- and expressed 
less IL-1ß, and the expression of anti-inflammatory genes, such as IL-10, was upregulated. Furthermore, treatment with BTK inhibitor increased the rate of phagocytosis by antiinflammatory M2 macrophages in vitro.

Conclusions Our findings show that BTK inhibition hinders M1 macrophage differentiation and skews monocytes towards an anti-inflammatory M2 phenotype, while enhancing apoptotic cell uptake by M2 cells. Therefore, BTK inhibition could have additional benefits in the treatment of autoimmune diseases such as SLE, by targeting both B cells and myeloid cells simultaneously.

Funding Source(s): This study was sponsored by EMD Serono Inc. (a business of Merck KGaA, Darmstadt, Germany)

\section{CLINICAL AND SEROLOGICAL PROFILE OF A SERIES OF RHUPUS PATIENTS}

${ }^{1}$ Beatriz Frade Sosa* ${ }^{2}$ Vera Ortiz-Santamaría, ${ }^{3}$ Vicente Torrente-Segarra, ${ }^{4}$ Ivan Castellvi, ${ }^{4}$ Berta Magallares, ${ }^{5}$ Delia Reina, ${ }^{6}$ Sonia Minguez, ${ }^{7}$ Meritxell Sallés, ${ }^{8}$ Sergi Ordoñez, ${ }^{9}$ Elena Riera, ${ }^{10}$ Maria Garcia Manrique, ${ }^{11}$ Jose A Gómez-Puerta. ${ }^{1}$ Rheumatology Department, Hospital Clinic, Barcelona; ${ }^{2}$ Hospital General de Granollers; ${ }^{3}$ Hospital Comarcal de l'Alt Penedès; ${ }^{4}$ Hospital Santa Creu i Sant Pau; ${ }^{5}$ Hospital Sant Joan Despi Moisès Broggi; ${ }^{6}$ AlTHAIA, Xarxa Assistencial Universitària de Manresa; ${ }^{7}$ ALTHAIA, Xarxa Assistencial Universitària de Manresa, Spain; ${ }^{8}$ Hospital Arnau de Vilanova, Lleida; ${ }^{9}$ Hospital Mutua de Terrassa; ${ }^{10}$ Hospital Parc Tauli, Sabadell; ${ }^{11}$ Rheumatology Department, Hospital Clinic, Barcelona, Spain

\subsection{6/lupus-2019-Ism.217}

Background Concomitant presence of two autoimmune diseases, such as rheumatoid arthritis (RA) and systemic lupus erythematosus (SLE) is known as Rhupus. Despite,

Abstract 217 Table 1 General characteristics of Rhupus and SLE patients

\begin{tabular}{|c|c|c|c|}
\hline & Rhupus ( $n=19$ ) & SLE $(n=38)$ & $P$ value \\
\hline Gender (Female), \% & $17(89.5)$ & $36(94.7)$ & 0.59 \\
\hline Mean age, years $\pm S D$ & $56.9 \pm 12.8$ & $45.9 \pm 12.3$ & 0.03 \\
\hline Disease duration, years $\pm S D$ & $13.9 \pm 7.0$ & $10.5 \pm 9.7$ & 0.24 \\
\hline Race (Caucasian), \% & $14(73)$ & $27(71.1)$ & 0.89 \\
\hline \multicolumn{4}{|l|}{ Clinical characteristics } \\
\hline Oral ulcers, $\%$ & $2(10.5)$ & $16(42.1)$ & $<0.01$ \\
\hline Articular involvement, \% & $19(100)$ & $36(94.7)$ & $<0.01$ \\
\hline - Arthritis, \% & $19(100)$ & $29(76.3)$ & 0.02 \\
\hline - Erosive disease, \% & $11(57.9)$ & $1(2.6)$ & $<0.01$ \\
\hline - Tenosynovitis, \% & $10(52.6)$ & $19(26.3)$ & 0.05 \\
\hline Leukopenia, \% & $3(15.8)$ & $21(55.3)$ & $<0.01$ \\
\hline Renal involvement, \% & $1(5.3)$ & $10(26.3)$ & 0.07 \\
\hline Mean SLEDAI * & $1.2 \pm 1.6$ & $3.3 \pm 3.4$ & 0.03 \\
\hline \multicolumn{4}{|l|}{ Immunological features } \\
\hline Mean RF levels, IU \pm SD & $184.6 \pm 199.3$ & $47.6 \pm 114.5$ & $<0.01$ \\
\hline Mean anti-CCP titers, IU \pm SD & $622.3 \pm 908.5$ & $5.1 \pm 5.2$ & $<0.01$ \\
\hline Positive anti-Ro antibodies, \% & $15(18.9)$ & $17(48.6)$ & 0.03 \\
\hline \multicolumn{4}{|l|}{ Treatment (ever) } \\
\hline Prednisolone, \% & $19(100)$ & $28(75.7)$ & 0.02 \\
\hline Methotrexate, \% & $17(89.5)$ & $13(36.1)$ & $<0.01$ \\
\hline Rituximab, \% & $8(44.4)$ & $5(14.7)$ & 0.04 \\
\hline
\end{tabular}

poliautoimmunity is not uncommon described in patients with systemic autoimmune diseases, only a small series of patients have been described so far with Rhupus. Our purpose was to analyze clinical and serological characteristics of patients with Rhupus and compare them with a cohort of patients with SLE.

Methods In this cross-sectional study, we included cases of Rhupus (RA-ACR/EULAR 2010 plus SLE-ACR 1987 criteria) from different Rheumatology Departments at Catalonia, Spain. In addition, we included patients with diagnosis of SLE in a 2:1 ratio matched by sex and race. All information was recorded following an established protocol.

Results A total of 57 patients were included, 19 cases with Rhupus and 38 cases of SLE alone as controls. 93\% of patients were female, Caucasian represented $71.4 \%$, Mestizo $17.9 \%$ and $5.4 \%$ were Asian. Mean age was $48.6 \pm 13.5$ years and mean disease duration was $11.48 \pm 9.1$ years. Main clinical characteristics were cutaneous involvement (75.0\%), hematological (66.0\%), serositis $(19.3 \%)$, renal disease $(17.9 \%)$ and secondary Sjögren syndrome (28\%) among others. Clinical and serological characteristics according groups are shown in table 1 .

Conclusions We found some clinical and serological differences among patients with Rhupus and SLE alone. As expected, articular domains and titers of RF and ACPAs were higher in Rhupus and they are more commonly treated with methotrexate and rituximab. By other hand, leukopenia, oral ulcers, anti-Ro antibodies and higher SLEDAI score were more common among SLE patients. Whether Rhupus patients represent a different condition requires further analysis in bigger cohorts.

\section{THERAPEUTIC TRAJECTORIES FOLLOWING HIGH DISEASE ACTIVITY STATE IN SLE}

${ }^{1}$ Christopher McMaster, ${ }^{1}$ Hieu Nim, ${ }^{1}$ Rachel Koelmeyer, ${ }^{2}$ Albert Frauman, ${ }^{3}$ Eric Morand, ${ }^{1}$ Alberta Y Hoi*. ${ }^{1}$ Monash University; ${ }^{2}$ Melbourne University; ${ }^{3}$ Monash University, Melbourne, Australia

\subsection{6/lupus-2019-Ism.218}

Background Systemic lupus erythematosus (SLE) is a heterogenous autoimmune disease. Treatment trajectories following high disease activity state (HDAS), as defined by SLEDAI score 10 , have not been well described.

Methods Longitudinal trajectories of patients from the Australian Lupus Registry were studied. HDAS periods were defined as the time from which HDAS begins, until the patient fulfils criteria for Low Lupus Disease Activity (LLDAS), or up to 365 days. Treatment escalation is defined as either an addition of hydroxychloroquine (HCQ), prednisolone (PNL) and immunosuppressant (IS), or any change in IS drug. De-escalation is either dose reduction or cessation of HCQ or IS without meeting treatment escalation criteria. Treatment trajectories were examined as the rolling sum (over time) of escalations and de-escalations and were clustered using k-means clustering methods. Different clustering partitions were tested. The $\mathrm{R}$ package $\mathrm{kml}$ was used for cluster determination and quality criterion calculations. The differences in time to resolution of HDAS between clusters were tested using likelihood ratio test. 